\title{
Towards Integrating Autonomously Created Knowledge Descriptions for the Semantic Web
}

\author{
Christian Mönch and Sari Hakkarainen \\ NTNU, Trondheim, Norway \\ \{moench, sari\}@idi.ntnu.no
}

\section{Creating Knowledge Representations for the Semantic Web}

In order for the Semantic Web to succeed, it has to be created in a decentralized way, analogous to the Web. In our model, the Semantic Web is composed by a number of individual entities. Every entity is an artifact, created by a creator. An entity contains a knowledge representation that contains knowledge about its associated resources. The creation of the knowledge representation is part of the creation of the entity. The knowledge representation is instantiated as an internal representation that is not visible to the outside, except for the creator of the entity.

Entities communicate by exchanging symbols. Symbols may be single words or more complex structures, e. g. RDF documents. Associated with each entity are two functions that allow to map between symbols and the internal representation of the knowledge:

1.) The interpretation function maps a symbol to the internal representation of the entity in a given time.

2.) The expression function maps a subset of the internal representation of an entity to symbols.

The interpretation and expression functions are depending on the structure and the state of the entities, i. e. on the internal representation. The interpretation function is parameterized by the background knowledge represented in an entity. The expression function is dependent of the symbols the creator chose for the represented knowledge, which is defined by the entity.

\subsection{The Problem of Heterogeneous Knowledge Representations}

Without continuous mutual agreement on different aspects of the knowledge representation heterogeneous knowledge representations will be created (cf. [1]). The reason is that the creator of an entity has to:

1.) select the part of their knowledge that should be represented in the entity, and

2.) select the granularity of the knowledge as well as the embedding of the knowledge into supporting structures.

The large number of individual decisions makes it very likely that different creators will create different entities and therefore differences in the expression and interpretation function, that are parameterized by the knowledge represented in the entity. As a result, different entities may map a given symbol on different concepts and/or identical symbols on different concepts. 
Since continuous mutual agreement on different aspects of the knowledge representation would be a conceptually centralized process, it can not be afforded and heterogeneous knowledge representations will emerge. In order for two entities to incorporate each others knowledge, the heterogeneity between them has to be resolved.

\section{On Demand Resolution of Heterogeneity}

If we want to allow the autonomous creation of knowledge representations - and that seems to be the only feasible way to create the Semantic Web- we need a strategy to cope with heterogeneity of knowledge descriptions. Our solution to an automated resolution is inspired by the mechanisms of discrepancy-resolution between persons. Individual persons acquire their knowledge in isolation. We can not decide whether our knowledge is identical —or compatible — because there is no cross-introspection. However, communication is used to detect and resolve differing interpretations of symbols. Individuals exchange symbols which they usually interpret in a compatible way, because they share a large amount of knowledge. But they may at some point of the communication experience a semantic mismatch and redirect their communication to disambiguate their interpretation of the exchanged symbols. In order to do so, the semantic mismatch has to be detected and quantified.

The heterogeneity makes it impossible to compare symbols directly in order to detect a semantic mismatch. Our solution is based on a, possibly repeated, verification step. Let us assume, entity $B$ interprets a symbol $S_{A}$ that was uttered by entity $A$. In order to verify its interpretation, entity $B$ asks entity $A$ for the validity of its interpretation. To do so, it utters its interpretation of symbol $S_{A}$ as the symbol $S_{B}$. This is then send to entity $A$, together with a symbol $S_{\text {context }}$ that describes the context of symbol $S_{B}$. Entity $A$ interprets $S_{B}$ using $S_{\text {context }}$, and calculates a distance value that tells entity $B$ how accurate its interpretation of symbol $S_{A}$ was.

The communication between the entities has to be grounded, i. e. based on symbols whose interpretation is by convention bound to one concept. In order to avoid a rigorous centralized definition process, we propose to use a large set of not exactly defined, but commonly understood symbols, e. g. WordNet. Of course there will be individual differences in the interpretation of the symbols. But these differences will be limited to individual subsets of the symbols. If we compare interpretations of a sufficiently large set of symbols, we will find a large number of compatible interpretations.

Measuring distances between parts of knowledge representations is difficult and not yet solved problem. Approaches for ontology comparison [2] might be applicable to this problem.

\section{References}

1. Mönch, C., Hakkarainen, S.: A Communication Based Approach to Integrate Decentraly Created Knowledge Descriptions. In: Proceedings of the WWW 12. (2003)

2. Mädche, A., Staab, S.: Measuring similarity between ontologies. In: Proceedings Of the EKAW-2002, Madrid, Spain, October 1-4, 2002. LNCS, Springer (2002) 